\title{
Using cranial electrotherapy stimulation to treat pain associated with spinal cord injury
}

\author{
Gabriel Tan, PhD, ABPP; ${ }^{1-2 *}$ Diana H. Rintala, PhD; ${ }^{1-2}$ John I. Thornby, PhD; ${ }^{1}$ June Yang, MD; $^{1-2}$ Walter Wade, \\ MD; ${ }^{1-2}$ Christine Vasilev, BS ${ }^{1}$ \\ ${ }^{1}$ Michael E. DeBakey Department of Veterans Affairs Medical Center, Houston, TX; ${ }^{2}$ Baylor College of Medicine, \\ Houston, TX
}

\begin{abstract}
Treatments for chronic pain in persons with spinal cord injury (SCI) have been less than effective. Cranial electrotherapy stimulation (CES), a noninvasive technique that delivers a microcurrent to the brain via ear clip electrodes, has been shown to effectively treat several neurological and psychiatric disorders. The present study examined the effects of daily 1-hour active CES or sham CES treatment (randomly assigned) for 21 days on pain intensity and interference with activities in 38 males with SCI. The active CES group $(n=18)$ reported significantly decreased daily pain intensity compared with the sham CES group $(n=20)$ (mean change: active CES = -0.73 , sham CES $=-0.08 ; p=0.03$ ). Additionally, the active CES group reported significantly decreased pain interference ( -14.6 pre- vs postintervention, $p=0.004)$ in contrast to the nonsignificant decrease in the sham CES group ( -4.7 pre- vs postintervention, $p=0.24$ ). These results suggest that CES can effectively treat chronic pain in persons with SCI.
\end{abstract}

Key words: adults, cranial, cranial electrotherapy stimulation, electric stimulation therapy, male, musculoskeletal, neuropathic, pain, rehabilitation, spinal cord injury, veterans.

\section{INTRODUCTION}

Chronic pain following spinal cord injury (SCI) is a significant issue that can affect the clinical outcome of postinjury rehabilitation and, ultimately, overall quality of life in numerous domains (e.g., physical, psychological, social, and occupational). At least two-thirds of persons with SCI experience frequent pain [1-3]. To date, the majority of studies on SCI pain have been conducted during initial rehabilitation or within the first few years after injury. However, most persons with SCI report chronic pain that persists and intensifies for many years following SCI [1]. Several temporal and circumstantially related patterns of SCI pain are notable (e.g., patient age, delayed rehabilitation, and localization of spinal insult), both in regard to the possible pathophysiological mechanisms involved and the potential implications for treatment and/or management [4-10]. Irrespective of these provocative or exacerbating factors, chronic pain after SCI has been shown to impose additional disability on a given degree of SCI-related disability [2,11-12]. Despite numerous experimental and clinical attempts at controlling chronic pain in persons with SCI, the vast majority of these treatments have been largely ineffective. Ragnarsson noted that the persistent refractoriness of chronic pain greatly reduces the resultant quality of life [13].

Abbreviations: ASIA = American Spinal Injury Association, BPI = Brief Pain Inventory, CES = cranial electrotherapy stimulation, MEDVAMC = Michael E. DeBakey Department of Veterans Affairs Medical Center, RA = research assistant, $\mathrm{SCI}=$ spinal cord injury, SD = standard deviation, VA = Department of Veterans Affairs.

* Address all correspondence to Gabriel Tan, PhD, ABPP; Pain Section, Anesthesiology (145), Michael E. DeBakey VA Medical Center, 2002 Holcombe Boulevard, Houston, TX 77030; 713-794-8794; fax: 713-794-7674.

Email: tan.gabriel@med.va.gov

DOI: 10.1682/JRRD.2005.04.0066 
Cranial electrotherapy stimulation (CES) is a noninvasive technique used for treating various conditions. The analgesic action of subperceptive levels of CES has been demonstrated in various clinical pain models [1415]. Extracellular recording techniques indicated that CES modifies noxious stimuli-evoked responses in the regions of the rat brain that are involved in nociceptive processing [16-17]. In humans, the mechanism of action of CES is not fully understood; however, it has been shown to stabilize neurotransmitter turnover [18], stimulate production of insulin growth factor- $1,{ }^{*}$ and facilitate normalization of monoamine levels following experimentally induced noxious stress. ${ }^{\dagger}$

Additionally, CES has been shown to enhance anesthetic effects in humans: CES increased nitrous oxide potency by approximately 37 percent [19] and reduced the required analgesic dose of fentanyl by approximately 33 percent in patients undergoing urologic surgery [20]. CES also has anxiolytic [21-22] and mood-enhancing effects [23] in humans. CES has been demonstrated to effectively decrease spinal [24], headache [25-27], dental [28-29], and muscle pain and spasms [30-32], as well as control several conditions often associated with pain (e.g., anxiety, depression, insomnia, and generalized stress) [33]. A recent double-blind, placebo-controlled study of fibromyalgia showed that CES was as effective as pharmacotherapy in reducing pain and, unlike pharmacotherapy, did not incur the risk of adverse side effects or the potential for polypharmacy [34]. In addition to pain reduction, CES also significantly improved the sleep, feelings of well-being, and reported quality of life of the patients with fibromyalgia. Donaldson et al. reported that CES modified the specific quantitative electroencephalographic "signatures" associated with fibromyalgia and this may have been partly responsible for, or reflective of, a significant decrease in pain and associated symptoms [35].

One advantage of CES is the apparent absence of adverse side effects. Prior to 1990, a few reports indicated mild burns at the electrode site [36], transient blurring of vision when electrodes were placed over the eyes

\footnotetext{
*Smith RB, Ryser CA. Important things we learn when research goes awry. In: Proceedings of the International Oxidative Medicine Association Conference; 2000 Aug 16-20; Denver, CO.

${ }^{\dagger}$ Gold MS, Pottash ALC, Sternbach H, Barbaban J, Asunitto W. Antiwithdrawal effect of alpha methyl dopa and cranial electrotherapy. Presented at the Annual Meeting of the Society for Neuroscience; 1982 Oct 31-Nov 5; Minneapolis, MN.
}

[37-42], slight dizziness [30], headache [43-44], giddiness [43], and tooth pain [43]. Such side effects appear to be related to the use of higher voltages and the placement of electrodes on the eyes. One should note that current iterations of CES consistently use lower voltage delivery and the method of placing electrodes over the eyes was discontinued nearly 30 years ago. More recently, Smith reported that of 23 psychiatric outpatients, 1 (4.3\%) cried during treatment and 1 (4.3\%) reported skin irritation behind the ears when the electrode gel began drying out [45]. In two postmarketing surveys (1995 and 1998), 47 physicians reported the results of CES use by 500 patients for various conditions [46]. Six patients (1.2\%) reported dizziness and two $(0.4 \%)$ reported nausea, both of which normally occur if the current is set too high. Three patients $(0.6 \%)$ reported skin irritation, and one each $(0.2 \%)$ reported anger, a metallic taste, a heavy feeling, and intensified tinnitus. Several studies conducted during the 1990s, with a combined total of 259 participants, reported no side effects that could reasonably be attributed to CES use [22-23,47-48].

Given the demonstrated success of CES in treating fibromyalgia, which involves a centrally mediated pain that is typically triggered by physical trauma (as is central neuropathic pain in persons with SCI), CES has been hypothesized to effectively reduce chronic pain after SCI and lower the burden of long-term pharmacologic management.

Capel et al. found that CES decreased the intensity of pain (of mixed etiology) and medication use in persons with SCI who received active CES treatment as compared with sham CES treatment [49]. In the first arm of the study, 14 participants received active CES and 13 received sham CES for 2 hours twice a day for 4 days. The active CES group reported less pain during and immediately after CES and reported that they used less pain medication. After an 8-week washout period, both groups received active CES for 2 hours twice a day for 4 days. The participants who had initially received sham CES showed significant improvement when they received active CES in the second arm of the study. Such results, while certainly encouraging, are somewhat limited because the mixed pain etiology in the investigation leaves unaddressed questions about the mechanistic basis of the effect(s). Furthermore, Capel et al.'s study used very small amounts of current $(12 \mu \mathrm{A})$, administered CES for a relatively long time each day (4 hours), and had a very short intervention period (4 days), which leaves 
unanswered questions about the optimal current, duration of daily treatment, and number of treatment days. In apparent recognition of these limitations, the authors concluded that larger studies of the effectiveness of CES for treating specific types of chronic pain in persons with SCI are needed [49].

Thus, given the potential of previous CES findings, together with the paucity of similar data on CES-based pain therapeutics in SCI, we undertook the present pilot study to assess the efficacy of CES on the intensity and specific behavioral correlates of musculoskeletal and neuropathic pain associated with SCI in a sample of veterans who had received care at a Department of Veterans Affairs (VA) SCI center. In addition, we explored the feasibility and logistics of having the participants self-administer the daily treatments in their homes over a 21-day period. This is particularly important given the mobility limitations that result from SCI and the need for adequate pain relief in the noninpatient care setting.

\section{METHODS}

\section{Participants}

A group of 40 veterans who were 6 months to 60 years post-SCI and with chronic musculoskeletal or neuropathic pain was recruited from the Michael E. DeBakey VA Medical Center (MEDVAMC) SCI Care Line in Houston, Texas. The inclusion criteria were-

1. Diagnosis of SCI with chronic pain of at least 3-months duration and moderate-to-severe intensity (i.e., a selfreported pain rating of 6 or above on a numeric scale from 0 to 10).

2. At least 6 months post-SCI.

3. Signed institutional-review-board-approved informed consent form.

4. Ability and willingness to comply with instructions, treatment regimen, and other study requirements.

5. Ability to travel to MEDVAMC two to three times, depending on group assignment.

The exclusion criteria were-

1. Documented history of noncompliance with past treatment or research studies (e.g., person missed follow-up appointments and did not take medications as directed).

2. Evidence of substance abuse (e.g., confirmed violation of medical orders, inappropriate dose escalation, procurement against medical advice, and/or current sub- stance abuse disorder as diagnosed by Diagnostic and Statistical Manual of Mental Disorders, Fourth Edition-Text Revision).

3. History of severe cognitive and/or mental disorder that might interfere with the treatment regimen.

Overall attrition was limited to two participants.

\section{Experimental Design}

The study used a double-blind, sham-controlled design with random assignment of participants to either an active CES or sham CES treatment group. The investigators, research assistant (RA), and participants were blinded to treatment type until the end of the initial phase.

\section{CES Equipment}

The CES equipment used was the Alpha-Stim ${ }^{\circledR} 100$ (Electromedical Products International Inc, Mineral Wells, Texas), a prescription medical technology that the U.S. Food and Drug Administration has approved for the management of pain, anxiety, depression, and insomnia. The Alpha-Stim ${ }^{\circledR} 100$ is illustrated in Figure 1.

Alpha-Stim ${ }^{\circledR}$ technology has been commercially available since 1981 and has been the subject of more than 50 studies to date. The unit uses microcurrent electrical therapy, and although a slight tingling sensation is sometimes felt under the electrodes, the observed/ reported treatment effect(s) is not contingent upon this liminal sensory input because many patients report complete absence of sensory stimulation with Alpha-Stim ${ }^{\circledR}$ 100 treatment. The therapy is delivered through easily applied ear clip electrodes. The manufacturer provided the CES units for the study.

Half the CES units delivered active CES and half delivered sham CES. Participants were unable to determine whether they were receiving active or sham CES, since the amount of electrical stimulation was set at a subthreshold level and could not be changed by the participants. The manufacturer also provided a third set of CES units for use in an open-label phase for those participants who were originally in the sham CES group. These units were the same as those available on the market; thus, the participant could adjust the stimulation level from 100 to $500 \mu \mathrm{A}$. The manufacturer trained the research staff on proper use of the CES units. 


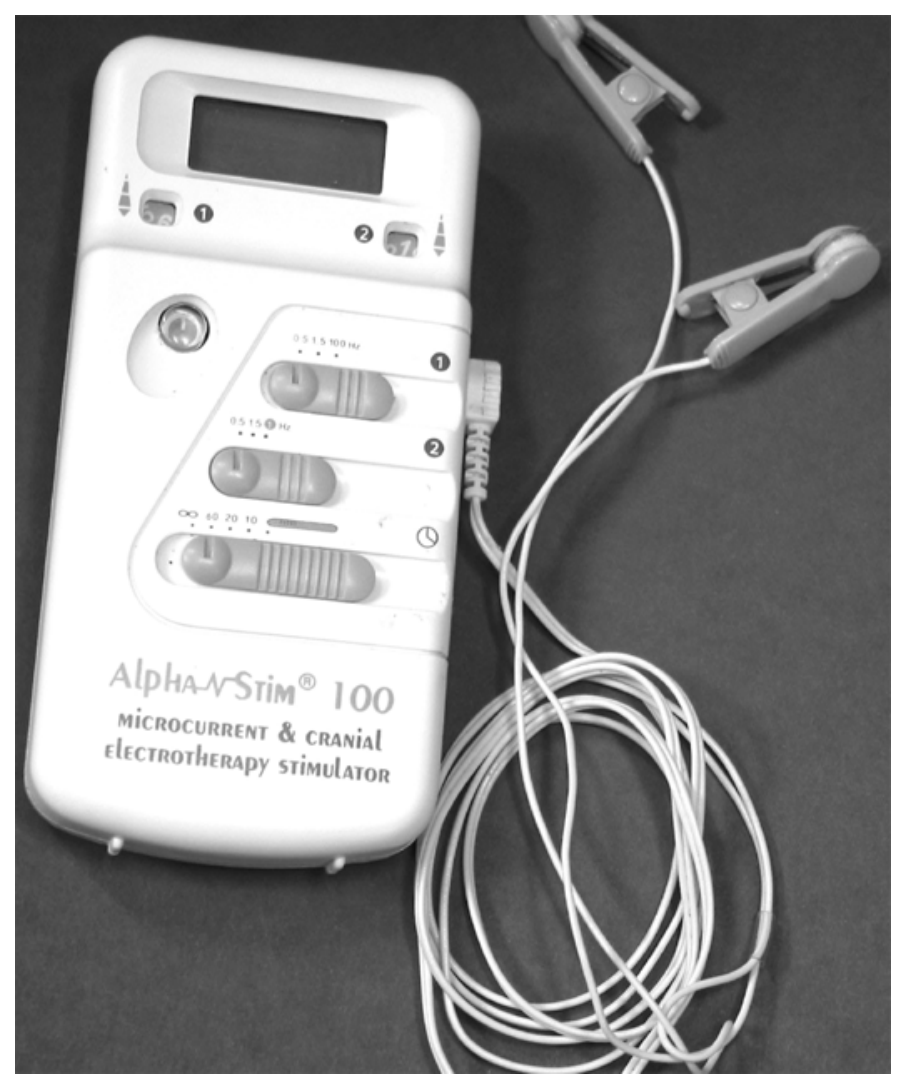

Figure 1.

Alpha-Stim ${ }^{\circledR} 100$ (Electromedical Products International Inc, Mineral Wells, Texas) cranial electrotherapy stimulation unit used in study.

\section{Procedures}

Participants were recruited by telephone from a list of patients with SCI who were on the registry at the MEDVAMC. Persons who agreed to participate met with the RA who explained the study, obtained informed consent, and explained the financial compensation that would assist with travel to the medical center (i.e., \$25 each data collection point). The RA also conducted a structured interview that included the preintervention questionnaire packet that is described in the "Measures" section. The RA instructed the participants in the selfadministration of CES and use of the daily pre- and postsession pain rating form. The participants were then randomly assigned to either the active or sham CES treatment groups. Participants in both groups received a CES unit to take home and were instructed to self-administer the treatment daily for 21 consecutive days. Participants who had the active CES units received 1 hour a day of $100 \mu \mathrm{A}$ subthreshold CES. The RA regularly con- tacted the participants by telephone, generally weekly, to answer questions and ensure that they were following study instructions.

After the initial 21-day trial, the participants returned to the clinic and completed a postintervention interview that included completion of a postintervention questionnaire packet. Then, the CES unit number was checked against a list (maintained by someone not directly involved in the study) for determining whether the unit had been programmed to deliver active or sham CES treatment. If the latter, the participant was offered the opportunity to participate in the open-label phase with an active CES unit. Those who chose to participate in the open-label phase were given the same instructions to selfadminister the CES treatment 1 hour a day and record their pain ratings immediately before and after each daily treatment session for another 21 consecutive days. Although these open-label units allowed the participants to adjust the level of current intensity at their own discretion, the participants were not required to record the intensity used. After the 21 days, the participants again returned to the MEDVAMC to complete another packet of questionnaires and return the CES unit.

\section{Measures}

We obtained demographic and injury-related information by self-report. These data included age, race/ ethnicity, educational status, marital status, date of SCI, and etiology of SCI.

We obtained the level and completeness of SCI from medical records. This data included the American Spinal Injury Association (ASIA) Impairment Scale grade, which indicates degree of completeness [50].

A physiatrist who cared for persons with SCI conducted a brief examination to determine whether the participants' chronic pain was neuropathic or musculoskeletal. Siddall et al. have proposed a three-tiered taxonomy for post-SCI pain [51-52]. The first tier categorizes pain as nociceptive or neuropathic based on accepted operational definitions of pain that reflect distinct peripheral and/or central mechanisms [53]. The second tier classifies nociceptive pain as being of musculoskeletal and/or visceral origin. The third tier classifies neuropathic pain according to site of occurrence or experience relative to the level of SCI.

Participants used daily pain rating forms to record pain immediately before and after each daily 1-hour treatment session. A numeric rating scale from 0 to 10 
was used; 0 indicated "no pain" and 10 indicated "pain as bad as you can imagine.”

As part of the preintervention questionnaire packet, we assessed pre- and postintervention pain intensity with the Pain Intensity subscale of the Brief Pain Inventory (BPI) [54]. Similar to the daily pain estimates, the BPI asked patients to rate on a 0 to 10 rating scale their pain: (1) "at its worst in the past 24 hours," (2) "at its least in the past 24 hours," (3) "on average," and (4) "right now." For each rating scale, 0 indicated "no pain" and 10 indicated "pain as bad as you can imagine."

Originally developed to assess cancer pain [54], the BPI was recently validated for the evaluation of nonmalignant chronic pain [55]. However, while the BPI has been used with persons diagnosed with chronic low-back and amputation pain [56-59], we are aware of no reports of the use of the BPI in patients with SCI. In the present study, Cronbach $\alpha$ for the BPI Pain Intensity subscale was 0.91 at preintervention and 0.92 at postintervention.

We assessed pre- and postintervention pain interference with a version of the Pain Interference subscale of the BPI that was modified for persons with physical disability [60]. This modified scale has 10 items that are rated on a 0 to 10 numeric rating scale. Participants were asked to rate the degree to which pain in the past week had interfered with 10 quality-of-life domains: general activity, mood, mobility, work, relations with other people, sleep, enjoyment of life, self-care, recreational activities, and social activities. For each item scale, 0 indicated that pain "does not interfere" and 10 indicated that pain "interferes completely." Data support the reliability and validity of the BPI for assessing pain interference in patients with cancer [54,61], and preliminary evidence supports the reliability of the modified Pain Interference subscale for assessing pain interference in patients with pain secondary to physical disability [60,62-63]. For the present study, Cronbach $\alpha$ for the BPI Pain Interference subscale was 0.95 at preintervention and 0.96 at postintervention.

\section{Data Analyses}

Descriptive statistics (mean, standard deviation [SD], and range for continuous variables; number and percentage for categorical variables) were obtained for each demographic and injury-related variable.

\section{Daily Pain Ratings}

We calculated each participant's average presession and postsession pain ratings (across the 21 daily sessions).
The difference between the presession and postsession average pain ratings was calculated and yielded one mean change score for each participant. We performed a twosample $t$-test to determine whether the average change score for the active CES group significantly differed from that of the sham CES group. Additionally, within each group (active, sham, and open-label), we performed paired $t$-tests to determine whether pain ratings significantly changed from pre- to postsession.

\section{Brief Pain Inventory Pain Intensity and Pain Interference}

For each data collection point (preintervention, postintervention, post-open-label), the 4 items on the BPI Pain Intensity subscale were summed for a composite pain intensity score and the 10 items on the BPI Pain Interference subscale were summed for a composite pain interference score. Change scores were calculated for each variable from pre- to postintervention for all 38 participants and from postintervention to post-open-label for the 17 participants in the open-label phase.

We conducted two-group t-tests to determine whether the active CES group pre- to postintervention change significantly differed from the sham CES group. Additionally, paired $t$-tests were performed separately for each group (active, sham, and open-label) for determining whether the individual subscale items and composite scores from pre- to postintervention were significantly different within each group.

\section{Exploratory Analyses}

We performed exploratory analyses ( $t$-test, analysis of variance, and Pearson correlation) to assess whether (1) etiology of SCI (traumatic or nontraumatic), (2) level and completeness of injury (tetraplegia with ASIA grade A, B, or C; paraplegia with ASIA grade A, B, or C; or tetraplegia/paraplegia with ASIA grade D), (3) type of pain (musculoskeletal or neuropathic), and/or (4) baseline levels of each measure were related to the amount of change in pain intensity or pain interference.

\section{RESULTS}

A total of 38 participants completed the study; 18 were randomly assigned to the active CES group and 20 to the sham CES group. The characteristics of the two groups are shown in Table 1. When participants in the 
JRRD, Volume 43, Number 4, 2006

Table 1.

Characteristics of participants with spinal cord injury (SCI) who received either sham $(n=20)$ or active $(n=18)$ cranial electrotherapy stimulation (CES).

\begin{tabular}{|c|c|c|c|c|}
\hline \multirow{2}{*}{ Characteristic } & \multicolumn{2}{|c|}{ Sham CES } & \multicolumn{2}{|c|}{ Active CES } \\
\hline & Mean \pm SD & Range & Mean \pm SD & Range \\
\hline$\overline{\text { Age (yr) }}$ & $56.6 \pm 10.9$ & $42-82$ & $56.0 \pm 8.3$ & $38-74$ \\
\hline \multirow{2}{*}{ Time Since SCI Onset (yr) } & $19.7 \pm 16.0$ & $<1-60$ & $20.1 \pm 10.3$ & $2-41$ \\
\hline & Number & Percent* & Number & Percent* \\
\hline Male & 20 & 100 & 18 & 100 \\
\hline \multicolumn{5}{|l|}{ Race/Ethnicity } \\
\hline White (non-Hispanic) & 13 & 65 & 12 & 67 \\
\hline African American & 4 & 20 & 5 & 28 \\
\hline Hispanic & 3 & 15 & 1 & 6 \\
\hline \multicolumn{5}{|l|}{ Educational Status (yr) } \\
\hline High School or Less & 7 & 35 & 4 & 22 \\
\hline Some College or More & 13 & 65 & 14 & 78 \\
\hline \multicolumn{5}{|l|}{ Marital Status } \\
\hline Married & 10 & 50 & 8 & 44 \\
\hline With Significant Other & 1 & 5 & 1 & 6 \\
\hline Neither & 9 & 45 & 9 & 50 \\
\hline \multicolumn{5}{|l|}{ Etiology of SCI } \\
\hline Traumatic & 18 & 90 & 15 & 83 \\
\hline Nontraumatic & 2 & 10 & 3 & 17 \\
\hline \multicolumn{5}{|l|}{ Level and Completeness of SCI } \\
\hline Tetraplegia (ASIA A, B, or C) & 4 & 20 & 4 & 22 \\
\hline Paraplegia (ASIA A, B, or C) & 10 & 50 & 6 & 33 \\
\hline All ASIA D & 6 & 30 & 8 & 44 \\
\hline \multicolumn{5}{|l|}{ Type of Pain } \\
\hline Neuropathic & 11 & 55 & 12 & 67 \\
\hline Musculoskeletal & 9 & 45 & 6 & 33 \\
\hline
\end{tabular}

Note: Sham and active CES groups did not significantly differ on any characteristics listed. Small cells were combined for chi-square analyses of race/ethnicity (white/nonwhite) and marital status (spouse or significant other/neither spouse nor significant other).

${ }^{*}$ Percentages may not sum to $100 \%$ because of rounding.

ASIA = American Spinal Injury Association (impairment classification A, B, C, D), SD = standard deviation.

sham CES group were later asked to participate in the open-label phase, 17 (85\%) agreed to do so.

\section{Daily Pain Ratings}

The active and sham CES groups did not differ significantly with regard to their average presession pain ratings (mean $=6.46$ active CES vs 6.08 sham CES). The two groups also did not differ significantly with regard to their average postsession pain ratings (mean $=5.73$ active CES vs 6.00 sham CES). However, the results of a two-sample $t$-test indicated that the average change in daily pain intensity from pre- to postsession was significantly larger for the active CES group (mean $=-0.73$ ) than the sham CES group (mean $=-0.08, p=0.03$ ). The treatment effect size was medium to large (Cohen $d=$ 0.76) as defined by Cohen [64]. Furthermore, the results of paired $t$-tests within each group indicated that participants who received sham CES did not show significantly reduced pain ( $p=0.34)$, whereas participants who received active CES did show significantly reduced pain $(p=0.02)$. In other words, the sham CES group's average daily postsession pain rating was 98.7 percent of the presession rating, whereas the active CES group's postsession rating was 88.7 percent of the presession rating. Finally, the 17 sham CES participants who subsequently participated in the open-label phase reported significant 
postsession pain reduction $(p=0.003)$. These findings are summarized in Table 2 .

Pain ratings before and after the daily treatment sessions for the active and sham CES groups are shown in Figures 2 and 3, respectively. Of the 20 individuals originally assigned to the sham CES group, 17 participated in the open-label phase. Mean pain ratings before and after daily treatment sessions for these 17 participants are displayed in Figure 4.

\section{Brief Pain Inventory}

\section{Pain Intensity}

Two-sample $t$-tests revealed that neither degree of change in any of the four BPI Pain Intensity subscale items nor the composite pain intensity score significantly differed between the active and Sham CES groups. However, paired $t$-tests within treatment groups revealed that among the four BPI Pain Intensity subscale items, the worst pain item decreased the most and therefore appeared most sensitive to CES. However, none of the changes in the BPI Pain Intensity subscale items was statistically significant for any of the three groups (active, sham, and open-label).

\section{Pain Interference}

Two-sample $t$-tests revealed no significant difference between the active and sham CES groups with regard to change from pre- to postintervention in any of the $10 \mathrm{BPI}$ Pain Interference subscale items or the composite pain interference score. However, in paired $t$-tests for the active CES group, 7 of the 10 individual Pain Interference subscale items significantly changed and reflected small to moderate effect sizes: general activity (Cohen $d=$ 0.67 ), self-care (Cohen $d=0.58$ ), sleep (Cohen $d=0.53$ ), social activities (Cohen $d=0.51$ ), normal work (Cohen $d=0.45$ ), enjoyment of life (Cohen $d=0.42$ ), and recreational activities (Cohen $d=0.38$ ). A paired $t$-test within the active CES group revealed that the composite pain interference score decreased significantly (mean change $=$ -14.6, $p=0.004$, Cohen $d=0.50$ ). For the sham CES group, neither the individual BPI Pain Interference subscale items nor the composite pain interference score changed significantly (mean change $=-4.7, p=0.24$ ); however, during the open-label phase, pain interference with sleep decreased significantly (Cohen $d=0.40$ ). The findings for the three BPI Pain Interference subscale items with effect sizes $>0.50$ and the composite pain interference score are displayed in Figures 5-8. Although the active CES group showed a significantly reduced composite pain interference score from pre- to postintervention, the change scores between the active and sham CES groups were not significantly different, partly because both groups showed decreased composite pain interference scores from pre- to postintervention. In other words, the slope for the active CES group was significant and the slope for the sham CES group was not significant; however, the two slopes were not significantly different from each other.

Exploratory analyses of the effectiveness of CES were performed only on the data from the initial active CES group $(n=18)$. The relationship of effectiveness to etiology of SCI, level and completeness of SCI, type of pain, and initial pain ratings was examined.

\section{Etiology of Spinal Cord Injury}

Only three participants in the active CES group had nontraumatic SCI. The mean change in daily pain ratings from pre- to postsession was -1.04 for the 3 participants with nontraumatic SCI and -0.67 for the 15 participants

Table 2.

Average daily pain ratings before and after 21-day cranial electrotherapy stimulation (CES). Participants rated pain on scale from 0 (“no pain”) to 10 ("pain as bad as you can imagine").

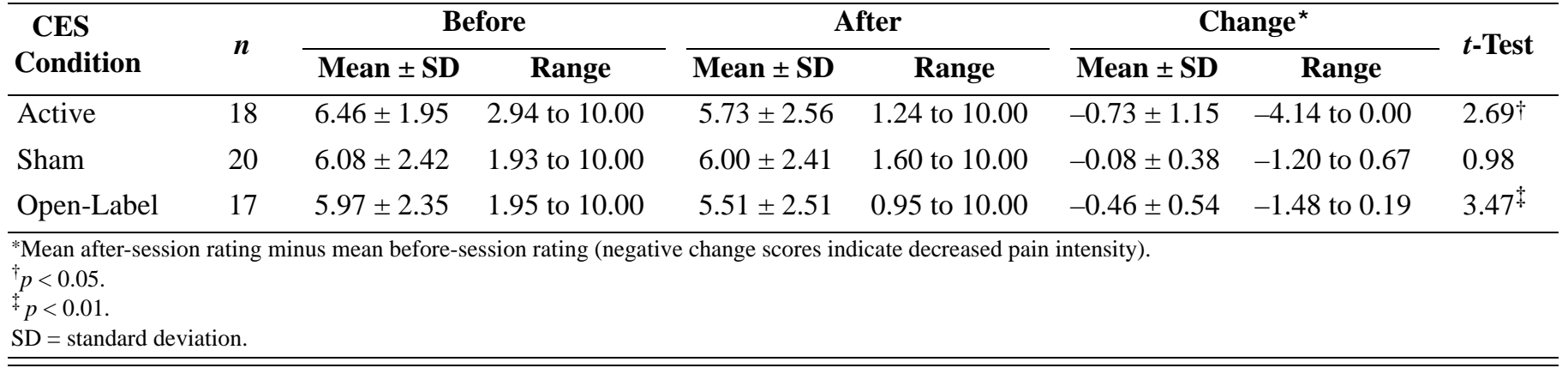




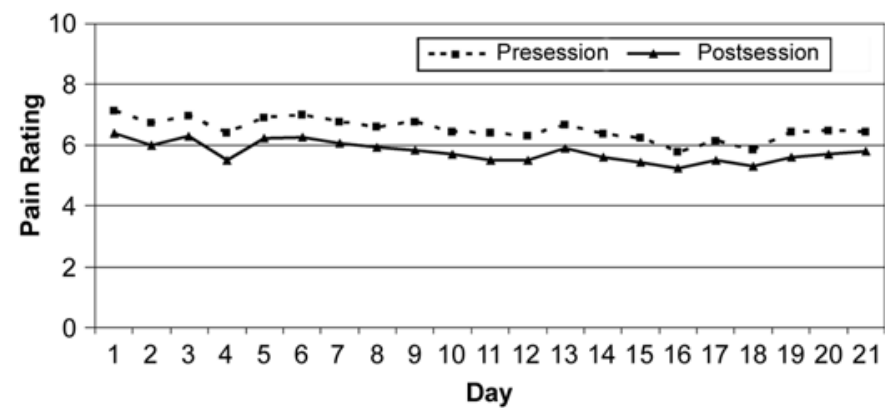

Figure 2.

Daily pre- and postsession pain ratings for participants with spinal cord injury who received active cranial electrotherapy stimulation ( $n=18$ ). Participants rated pain on scale from 0 ("no pain") to 10 ("pain as bad as you can imagine”).

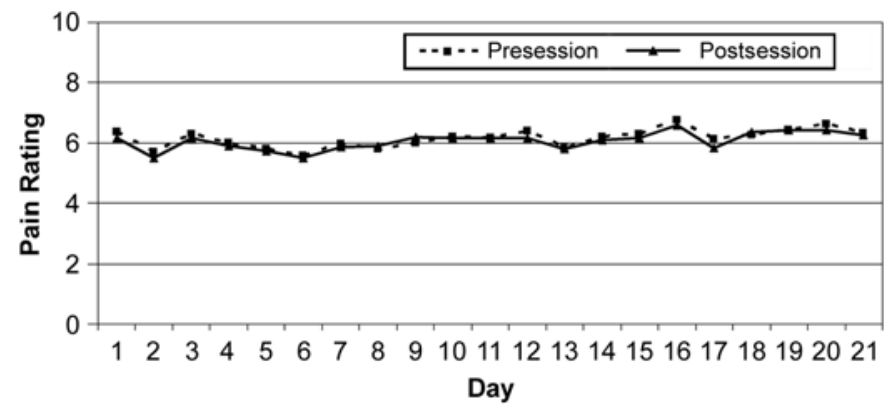

Figure 3.

Daily pre- and postsession pain ratings for participants with spinal cord injury who received sham cranial electrotherapy stimulation $(n=$ 20). Participants rated pain on scale from 0 ("no pain”) to 10 ("pain as bad as you can imagine”).

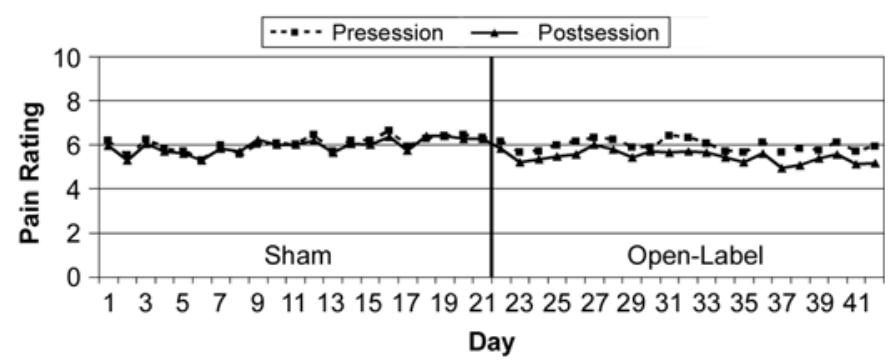

\section{Figure 4.}

Daily pre- and postsession pain ratings for 17 participants with spinal cord injury who received sham cranial electrotherapy stimulation (CES) in first arm of study and active CES in second (open-label) arm. Participants rated pain on scale from 0 ("no pain”) to 10 ("pain as bad as you can imagine”).

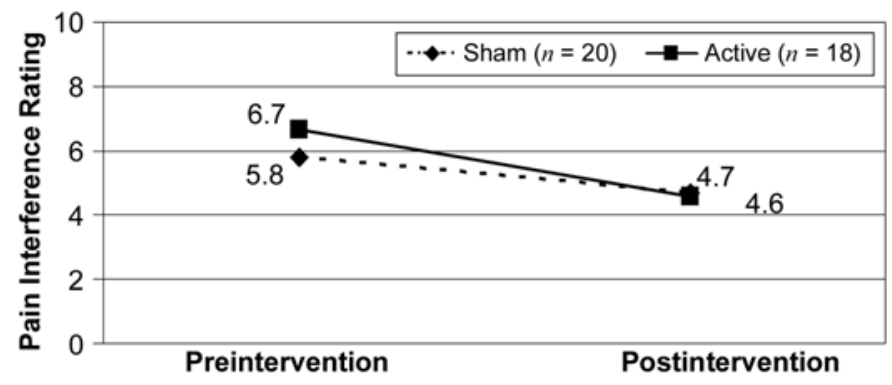

\section{Figure 5.}

Pre- and postintervention pain interference with general activity (as measured by Pain Interference subscale of Brief Pain Inventory) for participants who received either sham or active cranial electrotherapy stimulation. Participants rated pain interference on scale from 0 (“does not interfere”) to 10 (“interferes completely”).

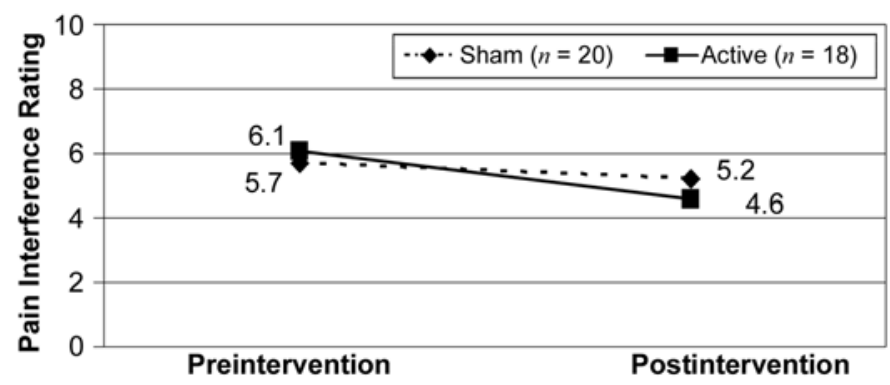

Figure 6.

Pre- and postintervention pain interference with enjoyment of life (as measured by Pain Interference subscale of Brief Pain Inventory) for participants who received either sham or active cranial electrotherapy stimulation. Participants rated pain interference on scale from 0 (“does not interfere") to 10 ("interferes completely").

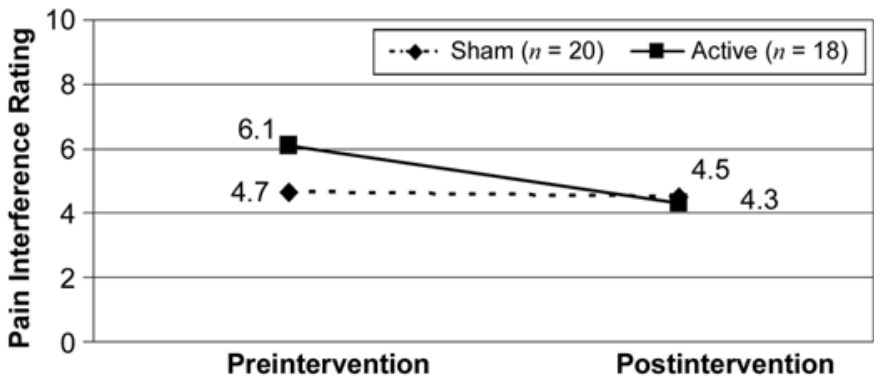

Figure 7.

Pre- and postintervention pain interference with social activities (as measured by Pain Interference subscale of Brief Pain Inventory) for participants who received either sham or active cranial electrotherapy stimulation. Participants rated pain interference on scale from 0 (“does not interfere”) to 10 (“interferes completely”). 




Figure 8.

Pre- and postintervention composite pain interference scores (total score for 10 items on modified Pain Interference subscale of Brief Pain Inventory) for participants who received either sham or active cranial electrotherapy stimulation.

with traumatic SCI. Decrease in pain intensity, as measured by the BPI Pain Intensity subscale, was greater for the nontraumatic vs traumatic SCI participants in the individual subscale items, as well as the composite pain intensity score (-2.33 vs -1.93 , respectively). Decrease in pain interference was also greater for the nontraumatic vs traumatic SCI participants for 5 of the 10 BPI Pain Interference subscale items; the two items that decreased significantly were enjoyment of life $(-5.33$ vs -0.73 , respectively, $p<0.001)$ and social activities ( -4.57 vs -1.20 , respectively, $p=$ $0.03)$; the composite pain interference scores were not significantly different (-21.33 vs -13.20 , respectively).

\section{Level and Completeness of Spinal Cord Injury}

In the active CES group, the mean difference between the daily pre- and postsession ratings was greater for those participants with less impairment from the SCI. Participants with tetraplegia with ASIA grade A, $\mathrm{B}$, or C $(n=4)$ had the smallest pain decrease (mean change $=-0.22$ ), participants with paraplegia with ASIA grade A, B, or C $(n=6)$ had a mean change of -0.46 , and participants with ASIA grade D $(n=8)$ had a mean change of -1.19 . This same pattern was observed for the average pain and worst pain items of the BPI Pain Intensity subscale. However, when change in pain interference was examined, participants with paraplegia with ASIA grade $\mathrm{A}, \mathrm{B}$, or $\mathrm{C}$ had the greatest decreases for 9 of the 10 individual BPI Pain Interference subscale items and the composite pain interference score.

\section{Type of Pain}

In the active CES group, 6 participants had musculoskeletal pain and 12 had neuropathic pain. Change in the daily pre- and postsession pain intensity ratings was larger for the neuropathic than the musculoskeletal group $(-0.81$ vs -0.57 , respectively); however, because of the small number of participants in each group, this difference was not statistically significant. The findings for pain intensity as measured by the BPI Pain Intensity subscale were mixed. Participants with musculoskeletal pain had greater pre- to postsession changes for two of the four items (worst pain and pain now) and the composite pain intensity score, while participants with neuropathic pain had greater pre- to postsession changes for the other two items (average pain and least pain). For pain interference, musculoskeletal pain improved more than neuropathic pain for 8 of the 10 BPI Pain Interference subscale items and the composite pain interference score $(-19.5$ vs -12.1 , respectively), but the differences were not significant.

\section{Relation of Change in Pain Ratings to Level of Initial Pain Ratings}

Examination of the relationship between the mean presession score and the mean daily change score revealed that change in pain during the sessions was greater for participants who had less intense presession pain, particularly those whose mean presession pain rating was 7 or less. However, when we examined the relationships between the preintervention BPI Pain Intensity subscale items and the change in pain intensity, participants with higher initial pain intensity had greater improvement in pain intensity during the 3-week study period. A similar pattern was found for the BPI Pain Interference subscale items and the composite pain interference score: participants with higher preintervention scores improved more during the 3-week period.

\section{DISCUSSION}

This study extended the knowledge base regarding effectiveness of CES for pain in persons with SCI. We established that persons with SCI can and will use the CES device at home for a 3-week period. This period of use is longer than that in other studies and this difference may be important. Some persons may need a longer trial period before experiencing any treatment effects. The technology of the specific CES device used in this study is quite different from the devices used in other studies. In this pilot study, the participants who received active CES reported, on average, significant pain reduction after each 
of the 21 daily sessions, but those who received sham CES did not (Figures 2-3), indicating a medium to large effect size. In separate paired $t$-tests, a significant difference was demonstrated between the pre- and postsession pain ratings for the active CES group but not the sham CES group. Furthermore, when the 17 participants originally in the sham CES group participated in the subsequent open-label phase, their pre- versus postintervention ratings significantly differed (Figure 4). The pain reduction in this study was not as great as in Capel et al.'s study in terms of pain intensity as a percentage of baseline [49]. In the first arm of their study, the pain rating on the final day was about 50 percent of baseline for the active CES treatment group, whereas our study found only an 11 percent change.

Many factors may allow the relatively small change in average daily pain ratings to be viewed in perspective. First, change scores varied considerably more in the active CES group than the sham CES group (SD $=1.15$ vs 0.38 , respectively; range $=4.14$ vs 1.20 , respectively). Second, we set the dose at a subthreshold of $100 \mu \mathrm{A}$ to maintain the double-blind design. Possibly, higher doses result in greater improvement in pain; however, to date, no study has examined the dose-response effect of CES on pain.

Post hoc exploratory examination of the daily pain rating data suggested that persons with nontraumatic SCI, lower level and/or less complete SCI, neuropathic pain, and mild-to-moderate pain intensity may get more immediate benefit from CES than persons with traumatic SCI, higher and/or more complete SCI, musculoskeletal pain, and more severe pain. Thus, the size of change in pain ratings likely increases when specific subgroups of persons with SCI are targeted for CES treatment. Additional studies are required for identifying the patients most likely to benefit from CES and for investigating the possible mechanistic basis of such effects. Studies are also needed for determining the duration of pain relief after each session. Figure 2 suggests that improvements in pain dissipated substantially by the next session. However, no known reason exists that CES treatment could not be used indefinitely every day.

The findings of paired $t$-tests within groups revealed that the observed reductions in the individual and composite BPI Pain Intensity subscale scores were not significant. However, the results showed that the composite pain interference score and several individual BPI Pain Interference subscale items decreased significantly for the active CES group but not for the sham CES group from pre- to postintervention.

Thus, these data indicate that CES effectively reduced pain intensity immediately after each treatment session, but its long-term effects on pain reduction were not statistically supported. Furthermore, exploratory analyses indicated that the group that benefited most from CES differed depending on the outcome measure examined (i.e., average daily decrease in pain intensity, 3-week decrease in pain intensity, or 3-week decrease in pain interference. Short-term relief of pain intensity was greater in persons with less severe pain, while longer term relief of pain intensity and interference was greater in persons with more severe pain. Such differences in effect may possibly be due to the small sample size. Future research should replicate the findings using a larger sample size and should more closely examine the issue of long-term treatment effects.

The fact that active CES significantly altered shortterm pain intensity and long-term pain interference but not long-term pain intensity should not compromise the importance of the findings. Other studies have shown that (1) pain interference plays a central role in mediating the relationship between negative emotions (such as depression) and disability [65], (2) pain interference mediates the effect of pain severity on depression [65], and (3) perceived control over pain interference with daily activities is more strongly associated with functioning than perceived control over pain intensity [66]. The relationships among pain intensity, pain interference, depression, and disability should be examined carefully in future studies on the effects of CES treatment.

One important limitation of the present study is that the participants were all male veterans who were receiving care at a VA healthcare facility. Generalizing the findings to female patients and other persons with SCI outside of the VA population should be done cautiously because of the somewhat unique sociodemographic factors inherent to our patient group and its type(s) and access to longitudinal care. Furthermore, this study did not address a number of important factors, including (1) the impact of CES on psychological distress and quality of life, (2) possible reduction in analgesic consumption, and (3) the amenability of patients to using the device long term if it were available. Finally, while many statistical tests were performed, thereby increasing the probability of chance findings, the preliminary nature of this study and the use of post hoc analysis of many variables was justifiable so 
as to better inform or suggest domains of inquiry for future studies.

\section{CONCLUSIONS}

In conclusion, the findings of this study, if replicated with larger samples, support the use of CES as a practical and effective treatment for particular types of SCIinduced pain.

\section{ACKNOWLEDGMENTS}

We wish to thank Daniel L. Kirsch, PhD, of Electromedical Products International, Inc (Mineral Wells, Texas) for the generous provision of Alpha-Stim ${ }^{\circledR} 100$ units for use in this study. Alpha-Stim ${ }^{\circledR}$ provided advice and consultation upon request from the authors. As well, we acknowledge the scientific advice and assistance of James Giordano, $\mathrm{PhD}$, on this article.

Dr. Yang is now with the Washington, DC, VAMC, and Ms. Vasilev is now with the University of Texas M. D. Anderson Cancer Center.

This material was based on work supported by the VA Rehabilitation Research and Development Service Center of Excellence on Healthy Aging with Disabilities, MEDVAMC, grant E0802-C.

The authors have declared that no competing interests exist.

\section{REFERENCES}

1. Siddall PJ, Loeser JD. Pain following spinal cord injury. Spinal Cord. 2001;39(2):63-73. [PMID: 11402361]

2. Rintala DH, Loubser PG, Castro J, Hart KA, Fuhrer MJ. Chronic pain in a community-based sample of men with spinal cord injury: prevalence, severity, and relationship with impairment, disability, handicap, and subjective wellbeing. Arch Phys Med Rehabil. 1998;79(6):604-14. [PMID: 9630137]

3. Rintala DH, Holmes SA, Fiess RN, Courtade D, Loubser PG. Prevalence and characteristics of chronic pain in veterans with spinal cord injury. J Rehabil Res Dev. 2005;42(5): 573-84. [PMID: 16586183]

4. Richards JS, Meredith RL, Nepomuceno C, Fine PR, Bennett G. Psycho-social aspects of chronic pain in spinal cord injury. Pain. 1980;8(3):355-66. [PMID: 7402693]
5. Davidoff G, Roth E, Guarracini M, Sliwa J, Yarkony G. Function-limiting dysesthetic pain syndrome among traumatic spinal cord injury patients: a cross-sectional study. Pain. 1987;29(1):39-48. [PMID: 3588000]

6. Rintala DH, Hart KA, Fuhrer MJ. Self-reported pain in persons with chronic spinal cord injury [abstract]. J Am Paraplegia Soc. 1991;14(2):83.

7. Burke DC. Pain in paraplegia. Paraplegia. 1973;10(4): 297-313. [PMID: 4697003]

8. Sie IH, Waters RL, Adkins RH, Gellman H. Upper extremity pain in the postrehabilitation spinal cord injured patient. Arch Phys Med Rehabil. 1992;73(1):44-48. [PMID: 1729973]

9. Summers JD, Rapoff MA, Varghese G, Porter K, Palmer RE. Psychosocial factors in chronic spinal cord injury pain. Pain. 1991;47(2):183-89. [PMID: 1762813]

10. Umlauf RL. Psychological interventions for chronic pain following spinal cord injury. Clin J Pain. 1992;8(2):111-18. [PMID: 1633374]

11. Tan G, Young S. Psychosocial and vocational issues in rehabilitation. In: Monga TN, Grabois M, editors. Pain management in rehabilitation. New York (NY): Demos Medical Publishing; 2002. p. 35-58.

12. Loubser PG, Donovan WH. Chronic pain associated with spinal cord injury. In: Narayan RK, Wilberger JE, Povlishock JT, editors. Neurotrauma. New York (NY): McGraw Hill; 1996. p. 1311-22.

13. Ragnarsson KT. Management of pain in persons with spinal cord injury. J Spinal Cord Med. 1997;20(2):186-99.

[PMID: 9144608]

14. Wilson OB, Hamilton RF, Warner RL, Johnston CM, deFriece R, Harter L, Schweitzer C, Talaverra J, Hymel CM, Skolnick MH. The influence of electrical variables on analgesia produced by low current transcranial electrostimulation of rats. Anesth Analg. 1989;68(5):673-81. [PMID: 2719297$]$ Erratum in: Anesth Analg. 1990;70(4):474.

15. Capel ID, Dorrell HM, Spencer EP. The application of subperception electrical stimuli elicits a temporally distinct response from restraint stress: I. Antinociceptive characteristics. J Bioelectricity. 1990;9:167-76.

16. Qiao JT, Skolnick MH, Dafny N. Dorsal raphe and external electrical stimulation modulate noxious input to single neurons in nucleus parafascicularis thalami. Brain Res Bull. 1988;21(4):671-75. [PMID: 3208154]

17. Dong WQ, Wilson OB, Skolnick MH, Dafny N. Hypothalamic, dorsal raphe and external electrical stimulation modulate noxious evoked responses of habenula neurons. Neuroscience. 1992;48(4):933-40. [PMID: 1630629]

18. Pozos RS, Strack LE, White RK, Richardson AW. Electrosleep versus electroconvulsive therapy. In: Reynolds DV, Sjoberg AE, editors. Neuroelectric research. Springfield (IL): Charles Thomas; 1971. p. 221-25. 
19. Stanley TH, Cazalaa JA, Limoge A, Louville Y. Transcutaneous cranial electrical stimulation increases the potency of nitrous oxide in humans. Anesthesiology. 1982;57(4):293-97. [PMID: 6982009]

20. Stanley TH, Cazalaa JA, Atinault A, Coeytaux R, Limoge A, Louville $Y$. Transcutaneous cranial electrical stimulation decreases narcotic requirements during neurolept anesthesia and operation in man. Anesth Analg. 1982;61(10):863-66.

[PMID: 7125252]

21. Braverman E, Smith R, Smayda R, Blum K. Modification of P300 amplitude and other electrophysiological parameters of drug abuse by cranial electrical stimulation. Curr Ther Res. 1990;48(4):586-96.

22. Krupitsky EM, Burakov AM, Karandashova GF, Katsnelson J, Lebedev VP, Grinenko AJ, Borodkin JS. The administration of transcranial electric treatment for affective disturbances therapy in alcoholic patients. Drug Alcohol Depend. 1991;27(1):1-6. [PMID: 2029855]

23. Philip P, Demotes-Mainard J, Bourgeois M, Vincent JD. Efficiency of transcranial electrostimulation on anxiety and insomnia symptoms during a washout period in depressed patients. A double-blind study. Biol Psychiatry. 1991; 29(5):451-56. [PMID: 2018818]

24. Kulkarni AD, Smith RB. The use of microcurrent electrical therapy and cranial electrotherapy stimulation in pain control. Clin Pract Altern Med. 2001;2(2):99-103.

25. Brotman P. Low-intensity transcranial electrostimulation improves the efficacy of thermal biofeedback and quieting reflex training in the treatment of classical migraine headache. Am J Electromed. 1989;6(5):120-23.

26. Solomon S, Elkind A, Freitag F, Gallagher RM, Moore K, Swerdlow B, Malkin S. Safety and effectiveness of cranial electrotherapy in the treatment of tension headache. Headache. 1989;29(7):445-50. [PMID: 2668227]

27. Romano TJ. The usefulness of cranial electrotherapy in the treatment of headache in fibromyalgia patients. Am J Pain Manag. 1993;3:15-19.

28. Clark MS, Silverstone LM, Lindenmuth J, Hicks MJ, Averbach RE, Kleier DJ, Stoller NH. An evaluation of the clinical analgesia/anesthesia efficacy on acute pain using the high frequency neural modulator in various dental settings. Oral Surg Oral Med Oral Pathol. 1987;63(4):501-5. [PMID: 3554095]

29. Hochman R. Neurotransmitter modulator (TENS) for control of dental operative pain. J Am Dent Assoc. 1988; 116(2):208-12. [PMID: 3278031]

30. Forster S, Post BS, Benton JG. Preliminary observations on electrosleep. Arch Phys Med Rehabil. 1963;44:481-89. [PMID: 14050721]

31. Magora F, Beller A, Aladjemoff L, Magora A, Tannenbaum J. Observations on electrically induced sleep in man. Br J Anaesth. 1965;37(7):480-91. [PMID: 5318569]
32. Kirsch DL, Lerner FN. Electromedicine: The other side of physiology. In: Weiner RS, editor. Innovations in pain management: a practical guide for clinicians. Boca Raton (FL): St. Lucie Press; 1998.

33. Kirsch DL, Smith RB. The use of cranial electrotherapy stimulation in the management of chronic pain: a review. NeuroRehabilitation. 2000;14(2):85-94. [PMID: 11455071]

34. Lichtbroun AS, Raicer MC, Smith RB. The treatment of fibromyalgia with cranial electrotherapy stimulation. J Clin Rheumatol. 2001;7(2):72-78.

35. Donaldson CCS, Sella GE, Mueller HH. The neural plasticity model of fibromyalgia: theory, assessment, and treatment. Pract Pain Manag. 2001;25-29.

36. Marshall AG, Izard CE. Cerebral electrotherapeutic treatment of depressions. J Consult Clin Psychol. 1974;42(1): 93-97. [PMID: 4814104]

37. Frankel BL, Buchbinder R, Snyder F. Ineffectiveness of electrosleep in chronic primary insomnia. Arch Gen Psychiatry. 1973;29(4):563-68. [PMID: 4748315]

38. Koegler RR, Hicks SM, Barger JH. Medical and psychiatric use of electrosleep. Transcerebral electrotherapy. Dis Nerv Syst. 1971;32(2):100-104. [PMID: 5313321]

39. Levitt EA, James NM, Flavell P. A clinical trial of electrosleep therapy with a psychiatric inpatient sample. Aust N Z J Psychiatry. 1975;9(4):287-90. [PMID: 769773]

40. McKenzie RE, Rosenthal SH, Driessner JS. Some psychophysiologic effects of electrical transcranial stimulation (electrosleep). In: Wulfsohn NL, Sances A, editors. The nervous system and electric currents. New York (NY): Plenum; 1976. p. 163-67.

41. Rosenthal SH, Wulfsohn NL. Electrosleep. A preliminary communication. J Nerv Ment Dis. 1970;151(2):146-51. [PMID: 5457619]

42. Rosenthal SH, Wulfsohn NL. Studies of electrosleep with active and simulated treatment. Curr Ther Res Clin Exp. 1970;12(3):126-30. [PMID: 4985490]

43. Singh K. Sleep inducing devices a clinical trial with a Russian machine. Int J Neuropsychiatry. 1967;3(4):311-18. [PMID: 6064118$]$

44. Matteson MT, Ivancevich JM. An exploratory investigation of CES as an employee stress management technique. J Health Hum Resour Adm. 1986;9:93-109.

45. Smith RB. Cranial electrotherapy stimulation in the treatment of stress related cognitive dysfunction, with an eighteen month follow up. J Cogn Rehabil. 1999;17(6):14-18.

46. Kirsch DL. Postmarketing survey of Alpha-Stim CES patients. In: Kirsch DL, editor. The science behind cranial electrotherapy stimulation. Edmonton (Canada): Medical Scope Publishing; 1999. p. 148.

47. Smith RB, Tiberi A, Marshall J. The use of cranial electrotherapy stimulation in the treatment of closed-head-injured patients. Brain Inj. 1994;8(4):357-61. [PMID: 8081350] 
48. Overcash SJ. A retrospective study to determine the effect of cranial electrotherapy stimulation (CES) on patients suffering from anxiety disorders. Am J Electromed. 1999; 16(1):49-51.

49. Capel ID, Dorrell HM, Spencer EP, Davis MW. The amelioration of the suffering associated with spinal cord injury with subperception transcranial electrical stimulation. Spinal Cord. 2003;41(2):109-17. [PMID: 12595874$]$

50. American Spinal Injury Association. International standards for neurological and functional classification of spinal cord injury. 5th ed. Chicago, IL: American Spinal Injury Association; 1996.

51. Siddall PJ, Yezierski RP, Loeser JD. Pain following spinal cord injury: clinical features, prevalence, and taxonomy. IASP Newsletter. 2000;(3):3-7.

52. Siddall PJ, Taylor DA, Cousins MJ. Classification of pain following spinal cord injury. Spinal Cord. 1997;35(2):69-75. [PMID: 9044512]

53. Giordano J. The neurobiology of nociceptive and anti-nociceptive systems. Pain Physician. 2005;8(3):277-90.

54. Cleeland CS. Measurement of pain by subjective report. In: Chapman CR, Loeser JD, editors. Issues in pain measurement. New York (NY): Raven Press; 1989. p. 391-403.

55. Tan G, Jensen MP, Thornby JI, Shanti BF. Validation of the Brief Pain Inventory for chronic nonmalignant pain. J Pain. 2004;5(2):133-37. [PMID: 15042521]

56. Small EJ, Smith MR, Seaman JJ, Petrone S, Kowalski MO. Combined analysis of two multicenter, randomized, placebo-controlled studies of pamidronate disodium for the palliation of bone pain in men with metastatic prostate cancer. J Clin Oncol. 2003;21(23):4277-84. [PMID: 14581438]

57. Lang E, Liebig K, Kastner S, Neundorfer B, Heuschmann P. Multidisciplinary rehabilitation versus usual care for chronic low back pain in the community: Effects on quality of life. Spine J. 2003;3(4):270-76. [PMID: 14589185]
58. Marshall HM, Jensen MP, Ehde DM, Campbell KM. Pain site and impairment in individuals with amputation pain. Arch Phys Med Rehabil. 2002;83(8):1116-19.

[PMID: 12161833]

59. Gammaitoni AR, Galer BS, Lacouture P, Domingos J, Schlagheck T. Effectiveness and safety of new oxycodone/ acetaminophen formulations with reduced acetaminophen for the treatment of low back pain. Pain Med. 2003;4(1): 21-30. [PMID: 12873275]

60. Tyler EJ, Jensen MP, Engel JM, Schwartz L. The reliability and validity of pain interference measures in persons with cerebral palsy. Arch Phys Med Rehabil. 2002;83(2):236-39. [PMID: 11833028]

61. Cleeland CS. Pain assessment in cancer. In: Osoba D, editor. Effect of cancer on quality of life. Boca Raton (FL): CRC Press; 1991. p. 293-305.

62. Engel JM, Schwartz L, Jensen MP, Johnson DR. Pain in cerebral palsy: the relation of coping strategies to adjustment. Pain. 2000;88(3):225-30. [PMID: 1068109]

63. Jensen MP, Ehde DM, Hoffman AJ, Patterson DR, Czerniecki JM, Robinson LR. Cognitions, coping and social environment predict adjustment to phantom limb pain. Pain. 2002;95(1-2):133-42. [PMID: 11790476]

64. Cohen J. Statistical power analysis for the behavioral sciences. 2nd ed. Hillsdale (NJ): Lawrence Earlbaum Associates; 1988.

65. Tan G, Thornby JI, Jensen MP. Revisiting negative emotions, pain, and functioning [abstract]. J Pain. 2004; 5(3 Suppl 1):124.

66. Tan G, Jensen MP, Robinson-Whelen S, Thornby JI, Monga T. Measuring control appraisals in chronic pain. J Pain. 2002;3(5):385-93. [PMID: 14622742]

Submitted for publication April 11, 2005. Accepted in revised form December 30, 2005. 